\title{
Notas micológicas XII: Stenocephalopsis subalutacea un discutido hongo hifomicetoso desde el aire de un Museo de Historia Natural deValparaíso (Chile)
}

\author{
Mycological notes XII: Stenocephalopsis subalutacea a discussed \\ hiphomycete fungus from the air of a Natural History Museum \\ (Valparaíso, Chile)
}

Eduardo Piontelli ${ }^{\text {* }}$, Anabell Lafuente², Peggy Vieille', Laura Carvajal'.

${ }^{1}$ Universidad de Valparaíso, Escuela de Medicina. Cátedra de Micología. Valparaíso, Chile.

${ }^{2}$ Museo de Historia Natural. Valparaíso

*Autor para correspondenica: eduardopiontelli@hotmail.com

RECIBIDO:05 de Diciembre de 2017

APROBADO:06 de Diciembre de 2017

DOI: 10.22370/bolmicol.2017.32.1.1035

LOS AUTORES DECLARAN NO TENER CONFLICTO DE INTERESES

Palabras claves: Stenocephalopsis subalutacea.

Key words: Stenocephalopsis subalutacea.

\section{RESUMEN}

En esta nota micológica, se comentan los aspectos taxonómicos, ecológicos y fisiológicos de la especie fúngica Stenocephalopsis subalutacea, la cual fue aislada desde el aire del museo de historia natural de Valparaíso, Chile. Se confirma su identificación mediante observación de las características morfológicas.

\section{INTRODUCCIÓN}

Stenocephalopsis subalutacea (Peck) Chamuris \& C.J.K. Wang, Mycologia 90 (3): 464 (1998) [MB\#443587]

Synonimos

$=$ Rhinotrichum subalutaceum Peck, Annual Report on the New York State Museum of Natural History 34: 51 (1880) [MB\#214686]

\section{ABSTRACT}

In this mycological note, we discuss taxonomical, ecological and physiological aspects of Stenocephalopsis subalutacea fungus. This species was isolated from the air of the Natural History Museum of Valparaíso, Chile. We confirms its identification through morphology characteristic observation.

=Basifimbria subalutacea (Peck) Hol.-Jech., Mycotaxon 14 (1): 141 (1982) [MB\#110502]

=Stenocephalum subalutaceum (Peck) Chamuris \& C.J.K. Wang, Mycologia 82: 531 (1990) [MB\#127716]

=Cephalosporium succineum Massee \& E.S. Salmon, Annals of Botany 16: 79 (1902) [MB\#218866] =Basifimbria succinea (Massee \& E.S. Salmon) Seifert \& W.B. Kendr. (1983) 
= Trichosporium ochraceum Am. Bull.. Soc.Mycol. Fr. 69: 270 (1953) nom. inval. (Art.36,7)

Los anamorfos de la División Ascomycota se encuentran entre los más numerosos integrantes de este gran grupo fúngico y su dispersión en muchos de los ambientes terrestres se efectúa principalmente desde el aire ${ }^{1,2}$. Este artículo, destaca la presencia en cultivo de un hongo de aparente distribución cosmopolita (nunca visto por los autores) por lo que se aportan sus datos para la literatura micológica chilena. Su aislamiento (solo una vez) se detectó desde el aire, en una rutina de muestreos semanales durante el año 2016 en el interior de las salas del Museo de Historia Natural de Valparaíso, mediante el método de decantación en placas petri conteniendo agar papa dextrosa. La sala del aislamiento llamada Parque Nacional La Campana (en el mes de Julio 2016), contenía 2 trozos aserrados de árboles desecados autóctonos, para su exhibición como muestras de la diversidad de los integrantes de ciertas zonas forestales del parque La Campana de la Región de Valparaíso.

El problema de la clasificación y diferenciación de algunos de los hongos anamórficos semejantes entre sí, no es un problema reciente, en especial cuando se emplea la clasificación basada solamente en la morfología ${ }^{3}$. Peck describe $R h i$ notrichum subalutaceum desde madera podrida en las montañas de Helderberg, New York State, en Noviembre de $1881^{4}$. Casi un siglo más tarde (1962), un raro hifomicete fue colectado por B. C. Lodha, en excremento de un caballo (pony) en las montañas Mussoorie, Uttar Pradesh, India. Stanley J. Hughes (1958), considera a esta especie, como un taxón un tanto relacionado a Rhinotrichum subalutaceum, pero posiblemente no idéntico ('affine') ${ }^{5}$. Posteriormente Subramanian \& Lodha (1968), describen un nuevo género y especie de hifomiceto: Basifimbria aurea para acomodar el hongo obtenido de excremento de caballo ${ }^{6}$. Hughes en 1978, describe $R$. subalutaceum desde madera muerta y corteza en Nueva Zelanda, comentando que esta especie obviamente 'to be redisposed'7.

Matsushima describe Rhinocladiella pe- ruviana, aislada en suelos de Los Andes, en Perú, en $1969^{\circ}$. De Hoog \& Hermanides-Nijhof en 1977 subsecuentemente transfieren esta especie a Basifimbria . Von Arx, sinonimiza Basifimbria, incluyendo $B$. aurea y $B$. peruviana y otros géneros caracterizados por sucesión simpodial de conidios rexolíticos en conidióforos ramificados, a Dicyma Boul. 1887, un género anamórfico unido a Ascotricha (Xylariales). ${ }^{10,11,23}$

Chamuris \& Wang, combinaron el epíteto de Rhinotrichum subalutaceum en Stenocephalum subalutaceum. Sin embargo, este nombre genérico no puede usarse debido a que se emplea como nombre genérico para un taxón de plantas de las Asteraceae y, debido a esto, los mismos autores transfieren la especie al nuevo género Stenocephalopsis $^{12}$. Estos autores accedieron a nuevas colecciones del mismo hongo desde desechos vegetales en el Estado de Nueva York, clasificándolo solo como un 'hyphomycete'. Basifimbria, fue descrita por Subramanian et Lodha, como género monotípico basado en la especie $B$. aurea aislada de excremento de caballo en la India y por ende una especie coprófila ${ }^{6}$. Holubová-Jechová (1982), considera $B$. aurea coespecífico con Rhinotrichum subalutaceum y hace la nueva combinación (Basifimbria subalutacea), un espécimen que se colectó varias veces en la República Checa, como así estudiando varios especímenes de otros países, demostrando que este hongo no solo es un coprófilo sino común en madera podrida, corteza, en tallos herbáceos cercanos al suelo y en hongos pyrenomycetosos, considerándolo probablemente un degradador herbáceo y de madera y una especie ampliamente dispersa ${ }^{13}$. Esta misma autora separa Hansfordia de $R$. subalutaceum a pesar de poseer una conidiogénesis similar.

Posteriormente, Hennebert, estudiando la conidiogénesis del hongo coprófilo Basifimbria aurea redescribe el género y lo considera a pesar de su variabilidad enfatizando la diferencia con Stenocephalopsis subalutacea ${ }^{14}$. Otra diferencia importante que este autor considera es la forma de sus células conidiógenas que son pálidas, ocrá- 
ceas y a menudo verrucosas en la parte inferior, «Abruptamente estrechada en el ápice» ${ }^{4}$ en una subhialina aparentemente lisa parte fértil cilíndrica cubierta de dentículos. Las células conidiógenas de Basifimbria aurea son completamente lisas, no se estrechan en el ápice pero a menudo es inflado, noduloso o geniculado, en la parte superior, característica más evidente cuando un cierto número de conidios se han formado. Los conidios que nacen a lo largo de la zona fértil de la célula conidiógena forman una elongada cabeza conidial en $S$. subalutacea, mientras que un irregular racimo de conidios se forma en $B$. aurea. La conidiogénesis aparece estrictamente simpodial y progresiva en S. subalutacea, pero ambiguamente progresiva y regresiva en $B$. aurea. Los dentículos son cortos y cilíndricos en $S$. subalutacea, mientras que son cilíndricos a cónicos, a veces alargados e inflados en $B$. aurea. Además, $S$. subalutacea es rexolítico, mientras $B$. aurea es rexolítico a esquizolítico. Las dos especies también difieren en sus hábitats naturales, S. subalutacea crece en madera de coníferas o madera dura, corteza, escombros de hierbas, musgos o helechos, mientras que $B$. aurea es exclusivamente coprófila ${ }^{14}$.

Al parecer, por la literatura analizada $S$. subalutacea tiene muchas similitudes morfológicas con varios géneros anamórficos dispersos en la literatura (Rhinotrichum, Basifimbria, Cephalosporium succineum, Trichosporium, Dicyma, Handsfordia) a veces difíciles de separar debido a las variaciones en cultivos ${ }^{10,13,15,16}$.

En la literatura actual, se describe que los anamorfos de ciertas trufas, Hydnocystis transitoria y H. bombycina (Ascomycota, Pezizomycotina, Pezizomycetes, Pezizomycetidae, Pezizales, Pyronemataceae) son sorprendentemente similares a las de Stenocephalopsis subalutacea ${ }^{12,17,18}$. Las similitudes incluyen color de la espora, conidios incrustados en gránulos y conidióforos, sobre células conidiógenas denticuladas que quedan en ápi- ces lisos después de la liberación de las esporas y patrones de ramificación y conidiación. Chamuris y Wang, también observaron que su anamorfo era similar a un integrante de los Pezizales como Geopyxis majalis ${ }^{12,19}$.

Basado en los análisis filogenéticos de $\mathrm{Ku}-$ mar et al. (2017), es dudoso que las especies de trufas en el linaje tarzetta-geopyxis se encuentren en múltiples continentes, excepto en los casos en que se hayan introducido debido a las actividades antropogénicas ${ }^{17}$. Podría ser interesante recolectar esporas similares de estas localidades y secuenciar el ADN de ellas para determinar si corresponden dentro del linaje tarzetta-geopyxis. Si este es el caso, puede ser una ayuda ecológica para la geografía y distribución de este grupo de hongos.

\section{Cultivo en PDA a $25^{\circ} \mathrm{C}$.}

A los 7 días, colonias pequeñas 10-13 milímetros, solevantadas granulosas, de colores ocráceos claros, reverso café oscuro. Conidióforos erectos, no ramificados, ramificados o verticilados, a veces ramificados dicotómicamente, divergentes y con un septo basal, entre 4-7 $\mu \mathrm{m}$ de ancho, de pared relativamente gruesa, de color ocre a cafesosos, lisos a verrucosos. Células conidiógenas, poliblásticas discretas simpodiales, pálidas a amarillo café más o menos lageniformes, a menudo verrucosas en la parte inferior, a veces elongadas, no septadas, $10-22 \times 4-5 \mu \mathrm{m}$ que se angostan a nivel del ápice, dando origen a una parte fértil cubierta por dentículos cortos y cilíndricos, no rugosos. Ameroconidios café pálidos, no en cadenas, redondos 7-9 $\mu \mathrm{m}$ en diám., a ligeramente ovados, 6-9 x $6-7,5 \mu \mathrm{m}$, secos, lisos a finamente rugosos, a veces con un apículo basal visible.

Hábitat.

En madera (mayormente angiospermas), Australasia, Europa, América del Norte ${ }^{16}$, Sudámerica (Chile). 

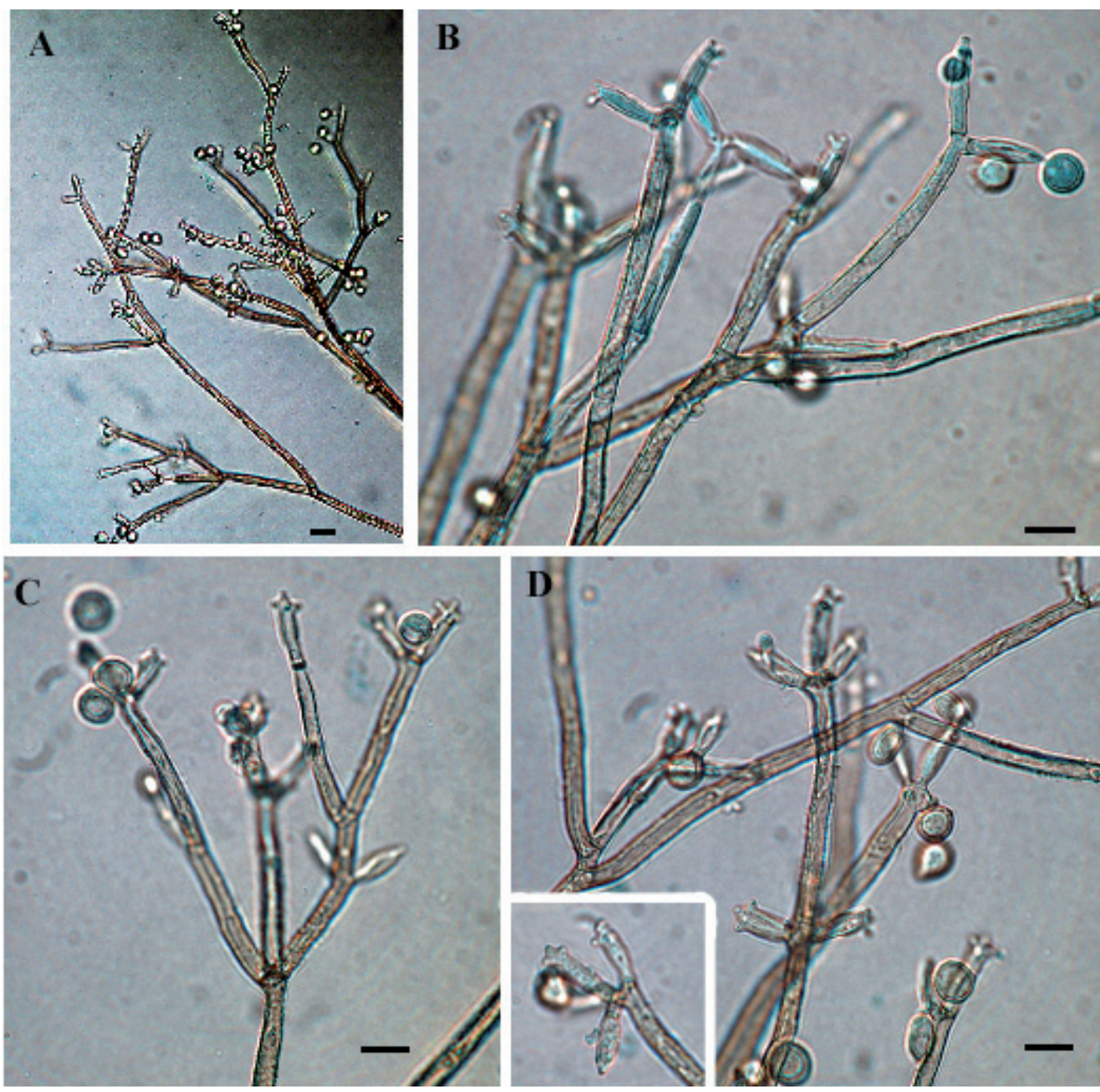

Figuras A-B-C-D. Stenocephalopsis subalutacea. A . Conidióforos ramificados y conidios a bajo aumento, B. Conidióforos jovenes, células conidiógenas con ápices angostos, denticulados y conidios, C-D. Conidióforos rugosos, células conidiógenas y conidios maduros (células conidiógenas denticuladas y rugosas en el recuadro). Barras $10 \mu \mathrm{m}$.

\section{CONCLUSIONES}

La clasificación morfofisiológica es el método que aún se emplea en el diagnóstico cuando no se ha aplicado la biología molecular en los hongos. Sin embargo, cuando la morfología se aplica sola, es común observar en ciertos géneros y especies la variabilidad en cultivo de las cepas analizadas, así como las posibles semejanzas en forma con otros taxa afines, como es nuestro caso. No hemos encontrado datos moleculares al respecto en la literatura. El CBS de Holanda tiene 2 cepas de $S$. subalutacea (CBS 564.97 Netherlands, CBS 670.92 NewYork ) sin secuenciación, mientras otras cepas dispersas en el mundo pueden estar en cultivos en diferentes centros o herbarios. Es interesante el trabajo actual de Kumar et al. (2017) y las similitudes de esta especie como anamorfo de ciertas Pezizales, pero faltan las comparaciones con los taxa existentes ${ }^{17}$. Es un hecho aparente, que esta especie no es coprófila como se comentó anteriormente $e^{4,7,12,13,14,20,21}$, sino que se encuentra en 
madera descompuesta de coníferas o madera dura, en corteza, como así en tallos herbáceos, musgos y helechos. Basifimbria aurea y B. spinosa provienen de excremento de oveja y de caballo, respectivamente. Nuestro aislamiento desde el aire de un museo de historia natural puede ser un hecho fortuito de dispersión anemófila, sin embargo, como posible coincidencia, la sala de su aislamiento contiene muestras en exhibición de trozos de árboles con corteza aserrados, así como otros integrantes vegetales de ciertas zonas forestales del país. Por las características morfológicas descritas confirmamos su identificación morfológica.

\section{REFERENCIAS}

1. Ibáñez V, Thompson L, Mañalich J. Fluctuación estacional de hongos anemófilos en Santiago Norte - Chile. Boletín Micológico. 1998; 13(12):47-56

2. Esquivel P, Mangiaterra M, Giusiano G, Sosa M. (2003). Microhongos anemófilos en ambientes abiertos de dos ciudades del Nordeste Argentino. Boletín Micológico. 2003; 18(1-2):21-28

3. Hennebert, G. L. Art. 59 and the problem with pleoanamorphic fungi. Mycotaxon. 1991; 40:479496

4. Peck, C. Report of the botanist. 34th Annual Report of the New York State Museum of natural History, Albany: University of the State of New York; 1881. Doc. No.127. p 24-58

5. Hughes, S.J. Revisiones Hyphomycetum aliquot cum appendice de nominibus rejiciendis. C. J. Bot. 1958; 36:727-838

6. Subramanian, C. V. \& Lodha, B. C. Two interesting coprophilous fungi from India. Current Science. 1968; 37(9): 245-248

7. Hughes, S. J. New Zealand Fungi. 25. Miscellaneous species. New Zealand Journal of Botany. 1978; 16(3): 311-370 DOI: 10.1080/0028825X.1978.10425143

8. Matsushima, T. Icones Microfungorum: a Matsushima lectorum. Kobe Japan: Matsushima, 1975
9. Hoog, G.S. de \& Hermanides-Nijhof. Survey of Black yeast an allied fungi. Study in Mycol. $1977 ; 15: 178-221$

10. Arx von, J. A. The Genera of Fungi: Sporulating in Pure Culture. J. Cramer, Vaduz; 1981

11. Arx von, J. A. The genus Dicyma, its synonyms and related fungi. Proceedings of the Koninglijke Nederlandse Akadademie Wettenschappen. 1982

12. Chamuris, G. P. \& Wang, C. J. K. Stenocephalum, a new genus for Rhinotrichum-subalutaceum (Hyphomycetes). Mycologia. 1990; 82: 528-532.

13. Holubová-Jechová, V. Some notes on Basifimbria aurea. Mycotaxon. 1982; 14(1):140-144

14. Hennebert, G.L. Diagnosis and variability of coprophilous Basifimbria aurea. Mycol. Res. 2005; 109(5): 595-602

15. Masse, G \& Salmon, E.S. Researches on coprophilous fungi.II. Annals of Botany. 1902; 16(61):57-93

16. Seifert, K et al. The genera of Hyphomycetes-2011 update. Persoonia. 2011; 27: 119-129

17. Kumar, M. L., Smith, E.M., Nouhura, R.E. et al. A Molecular and morphological re-examination of the generic limits of truffles in the tarzetta-geopyxis 
lineage-Densocarpa, Hydnocystis, and Paurocotylis. Fungal Biology. 2017; 121(3):264-284.

18. Chamuris, G. P. \& Wang, C. J. K. Stenocephalopsis, not Stenocephalum as the genus for Rhinotrichum subalutaceum. Mycologia. 1998; 90(3): 464.

19. Paden, J.W. Imperfect states and the taxonomy of the Pezizales. Persoonia. 1972; 6(4): 405-414.

20. Saccarrdo, P.A. Fungi veneti nuovi vel critici V. Mycologicae Venetae addendi. Michelia. 1881a; 2:241-301
21. Saccarrdo, P.A. Fungi gallici lecti a cl. viris. P. Brunaund, C.C. Gillet, Abb. Letendre, A. Malbranche, J. Therri valcditi in Mycoteca Gallica Cl. C. Roumegueri. Michelia. 1881b; 2:302-383

22. Ross Sumstine. D. Studies in North American Hyphomycetes: I. Mycologia. 1911; 3(2):45-56

23. Hawksworth, D. L. A revision of the genus Ascotricha Berk. Mycological Papers. Leipzig, Alemania: Kew, Commonwealth Mycological Institute; 1971 